\title{
Does last contraceptive method used impact the return of normal fertility?
}

\author{
Yes, according to a prospective observational study \\ of more than 17,000 women that evaluated fecundability \\ after stopping contraception. The authors found hormonal \\ intrauterine device (IUD) users had slightly increased \\ fecundability compared with users of barrier methods. \\ There was no difference in fecundability for users of copper \\ IUDs, implant, oral contraception, patches, rings, \\ or natural methods compared with barrier methods. Users \\ of injectable contraceptives experienced the longest delay \\ in return of normal fertility, about 5 to 8 menstrual cycles.
}

Yland JJ, Bresnick KA, Hatch EE, et al. Pregravid contraceptive use and fecundability: prospective cohort study. BMJ. 2020;371:m3966.

\section{EXPERT COMMENTARY}

Lisa Hofler, MD, MPH, MBA, is Chief, Division of Family Planning, Department of Obstetrics and Gynecology, University of New Mexico, Albuquerque.

Lindsay Dale, MD, is Fellow in Complex Family Planning, Division of Family Planning, Department of Obstetrics and Gynecology, University of New Mexico, Albuquerque.

effect of recent

contraceptive

use on fertility

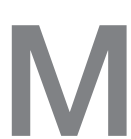

ost US women aged 15 to 49 currently use contraception, with long-acting reversible contraception (LARC)-IUDs and the contraceptive implant-increasing in popularity over the last decade. ${ }^{1}$ Oral contraceptive pills, male condoms, and LARC are the most common reversible methods used. ${ }^{1}$ While the efficacy and safety of contraception have been established, few studies have examined the effect of recent contraceptive use on fertility.

The authors report no financial relationships relevant to this article.

doi: 10.12788/obgm.0060
Fecundability is the probability of pregnancy during a single menstrual cycle for a couple engaging in regular intercourse and not using contraception. ${ }^{2}$ Small studies have found short-term reductions in fecundability after discontinuing combined oral contraceptives and larger reductions after stopping injectable contraceptives, with no long-term differences among methods. ${ }^{3,4}$

Data are limited regarding the effects of other forms of contraception on fecundability, particularly LARC methods. A recent study was designed to evaluate the association between the last contraceptive method used and subsequent fecundability. ${ }^{2}$

\section{Details of the study}

Yland and colleagues pooled data from 3 prospective cohort studies of 17,954 women planning pregnancies in Denmark, Canada, and the United States. Participants reported the contraceptive method used most recently before trying to conceive. They completed questionnaires every 2 months for 12 months or until they reported a pregnancy. Women were excluded if they tried to conceive for more than 6 menstrual cycles at study entry. 
The authors calculated the fecundability ratio-the average probability of conception per cycle for a specific contraceptive method compared with a reference method-using proportional probability models adjusted for potential confounders. They also calculated pregnancy attempt time using participant-reported menstrual cycle length and date of last menstrual period during follow-up questionnaires.

\section{Injectable contraceptives associated with longest delayed fertility return}

After adjusting for personal factors, medical history, lifestyle characteristics, and indicators of underlying fertility, the authors found that injectable contraceptive use was associated with decreased fecundability compared with barrier method use (fecundability ratio $[\mathrm{FR}], 0.65 ; 95 \%$ confidence interval [CI], 0.47-0.89). Hormonal IUD use was associated with slight increases in fecundability compared with barrier method use (FR, 1.14; 95\% CI, 1.07-1.22) and copper IUD use (FR, 1.18; 95\% CI, 1.05-1.33). All other contraceptive methods were not significantly different from barrier methods.

LARC method use was associated with the shortest delay in return of normal fertility ( 2 cycles), followed by oral and ring contraceptives (3 cycles) and patch (4 cycles). Women using injectable contraceptives experienced the longest delay (5-8 menstrual cycles). Lifetime duration of contraceptive use did not impact fecundability in the North American cohort.

\section{Study strengths and limitations}

This large, prospective study contributes useful information about fecundability after stopping contraceptive methods. It confirms earlier studies' findings that showed decreased fecundability after stopping injectable contraceptives. Study participants' most recent method used was similar to overall US method distribution. ${ }^{1}$

\section{WHAT THIS EVIDENCE MEANS FOR PRACTICE}

This is the largest study to date to evaluate fecundability after stopping different contraceptive methods among women planning pregnancies. The study confirms previous research that associated injectable contraceptives with delayed return of normal fertility. It provides reassurance for counseling users of IUDs, implants, oral contraception, ring, and patch: those methods were not associated with reduced fecundability compared with barrier methods. The study also suggests long-term contraceptive use does not decrease fecundability.

Women may ask when to stop their contraceptive method to optimally time a pregnancy. In this study, measurements of return to normal fertility were imprecise. Individualized counseling, accounting for personal circumstances, is still best when advising when to stop contraception for couples planning pregnancy.

LISA HOFLER, MD, MPH, MBA, AND LINDSAY DALE, MD

Study limitations include online recruitment of self-selecting participants, which introduces selection bias. The study population was overwhelmingly white $(92 \%)$ and highly educated ( $70 \%$ with college degrees), quite different from the US population. These findings may therefore have limited generalizability. Additionally, injectable contraceptive users had higher body mass index and were more likely to smoke and have diabetes, infertility, or irregular menstrual cycles. IUD users were more likely to be parous and have a history of unplanned pregnancy, indicating possible higher baseline fertility. Even after adjusting, possible unmeasured factors could impact study results.

\section{References}

1. Daniels K, Abma JC. Current contraceptive status among women aged 15-49: United States, 2017-2019. NCHS Data Brief, no. 388. Hyattsville, MD: National Center for Health Statistics; 2020.

2. Yland JJ, Bresnick KA, Hatch EE, et al. Pregravid contraceptive use and fecundability: prospective cohort study. BMJ. 2020;371:m3966.

3. Hassan MA, Killick SR. Is previous use of hormonal contraception associated with a detrimental effect on subsequent fecundity? Hum Reprod. 2004;19:344-351.

4. Mansour D, Gemzell-Danielsson K, Inki P, et al. Fertility after discontinuation of contraception: a comprehensive review of the literature. Contraception. 2011;84:465-477.

\begin{tabular}{l}
\hline FAST \\
TRACK \\
Injectable \\
contraceptive use \\
was associated \\
with decreased \\
fecundability \\
compared with \\
barrier method \\
use; hormonal \\
IUD use was \\
associated with \\
slight increases \\
in fecundability \\
compared with \\
barrier method \\
use and copper \\
IUD use
\end{tabular}

